\title{
Signal setting in an urban area: a procedure with day-to-day dynamic and stability constraints
}

\author{
G. E. Cantarella ${ }^{1}$, P. Velonà ${ }^{2} \&$ A. Vitetta ${ }^{2}$ \\ ${ }^{1}$ Dept of Civil Engineering, University of Salerno, Italy \\ ${ }^{2}$ Dept of Maths, Comp. Sci., Electr., Transp., \\ Mediterranean University of Reggio Calabria, Italy
}

\begin{abstract}
In this paper a model and a procedure for signal setting design with demand assignment are reported. The model is developed within a day-to-day dynamic framework where demand assignment is dealt with through deterministic (or stochastic) process models. The system of models, based on a what-to approach, generates signal timings taking into account users path choice behaviour as well as stability constraints. The main contribution in this paper is the specification of the heuristic procedure for signal setting optimization, based on genetic algorithm. The procedure is tested on a real scale test site with the objectives to validate the system of models and the optimization procedure. A sensitivity analysis with respect to demand level is also briefly discussed.
\end{abstract}

Keywords: signal setting, traffic control, day-to-day dynamic.

\section{Introduction}

This paper deals with the design of signal setting in urban areas. This problem can not be solved without considering the user behaviour at least at route choice level. The resulting problem belongs to the class of problem of transportation supply design with assignment [1-4].

Two main approaches have been proposed with respect to the method for demand assignment (with probabilistic path choice behaviour): equilibrium assignment, effectively approached by fixed-point models; day-to-day dynamic process models, derived from time-discrete non-linear dynamic system theory. 
Three main approaches have been proposed with respect to the method for signal setting design: with fixed flows, with flows from equilibrium assignment, with flows from day-to-day dynamic assignment.

In signal setting with fixed flows, say optimization of signal timings, the flow pattern is considered known (for a general review see [5]). This approach can be considered day-to-day static because the flows (obtained from observations or from a model) are supposed fixed as regard the optimization in each time slice, even though they can vary during consecutive time slice.

In signal setting with equilibrium assignment, the flow pattern is considered affected by the user path choice behaviour. Interaction between the user choice behaviour and the level of service provided by the transportation network is addressed through user equilibrium assignment that searches for mutually consistent arc flows and costs. Signal setting with equilibrium assignment has been proposed in literature since middle 70s [6] according to exact approaches, say Global Optimization with Equilibrium (EGO), or heuristic approaches, such as Recursive Optimization (RO) (also called iterative procedures) and Embedded Optimization (EO). Some methods are relative to the signal setting design in emergency condition $[7,8]$.

In signal setting with day-to-day assignment, the evolution over time of the flow pattern is explicitly modelled through dynamic process models.

The approaches proposed in literature are based fixed flows or on equilibrium assignment, well established in literature. Some relevant issues may not be effectively addressed under the equilibrium approach, mainly uniqueness and stability, sensitivity to parameters and/or starting state. These issues are better casted within the day-to-day dynamics paradigm, including deterministic process models as well as stochastic ones.

In this paper a model and a procedure for signal setting design are reported. The model is developed within a day-to-day dynamic framework where demand assignment is dealt with through deterministic process models. This problem seems relevant since optimization of signal timings under equilibrium assumptions may not guarantee that an effective solution is obtained, because it may well be not an attractor of the evolution over time.

The main contribution of this paper is the specification of the general procedure, based on a genetic algorithm, specified and applied to a real size network for solving the described model. Results of a sensitivity analysis with respect to demand level are also reported.

After this introduction, section 2 briefly reports the main part of the general model that is reported in [9]. Section 3 reports the genetic proposed procedure specified for the optimization model. Section 4 describes how the proposed approach can be applied to real scale networks and some sensibility of the procedure in relation to the user choice simulation behaviour. Section 5 reports some conclusions and further developments. 


\section{Models for signal setting}

A transportation system is usually modelled through a network with a transportation cost $c_{a}$ and a flow $f_{a}$ associated to each arc $a$. Without any loss of generality each access $a$ of a junction is assumed corresponding to arc $a$ (but not necessarily vice versa). Users are assumed grouped into classes. Let (in alphabetical order):

$\mathbf{B}_{\mathrm{i}} \quad$ is the arc-path incidence matrix for user class $i$, with entries $b_{a k}=1$ if arc $a$ belongs to path $k, b_{a k}=0$ otherwise;

c be the vector of arc costs, with entries $c_{a}$, including delays at junctions, running times, monetary costs for tolls, etc.;

$d_{i} \geq 0$ be the demand flow for users belonging to class $i$;

$\mathbf{f} \geq \mathbf{0}$ be the vector of arc flows, with entries $f_{a}$; hence for an arc $a$ corresponding to an access $f_{a}$, is the arrival flow;

$\mathbf{f}^{\mathrm{t}} \quad$ be the vector of arc flows at day $t$;

$\mathbf{g} \in S_{g}$ be the vector of signal timings (such as green times, cycle time, starting or ending time of the green period for each access, the offset of the signal plan for each junction, and possibly others);

$\mathbf{x}^{\mathrm{t}}$ be the vector of arc forecasted costs at day $t$, say the costs that affect today user choice behaviour;

$n$ be the number of arcs;

$\mathbf{p}_{\mathrm{i}} \geq \mathbf{0}$ is the vector of path choice probabilities for user class $i$, with $\mathbf{1}^{\top} \mathbf{p}_{\mathrm{i}}=1$, with entries given by the probability $p_{k}$ that users of class $i$ choose path $k$;

$S_{f}=\left\{\mathbf{f}=\sum_{\mathrm{i}} d_{i} \mathbf{B}_{\mathrm{i}} \mathbf{p}_{\mathrm{i}}: \mathbf{p}_{\mathrm{i}} \geq \mathbf{0}, \mathbf{1}^{\top} \mathbf{p}_{\mathrm{i}}=1 \forall i\right\}$ be the set of feasible arc flows, nonempty (if the network is connected), compact, convex;

$S_{g}$ be the set of feasible signal timings (including non-negativity constraints).

\subsection{Static flows}

Congestion is generally simulated assuming that arc costs depend on arc flows, and possibly on signal timings through the arc cost function:

$$
\mathbf{c}=\mathbf{c}(\mathbf{f}, \mathbf{g})
$$

The arc flow function can be defined as:

$$
\mathbf{f}=\mathbf{f}(\mathbf{c}) \sum_{\mathrm{i}} d_{i} \mathbf{B}_{\mathrm{i}} \mathbf{p}_{\mathrm{i}}\left(-\mathbf{B}_{\mathrm{i}}^{\top} \mathbf{c}\right) \in S_{f}
$$

The user equilibrium assignment searches for mutually consistent arc flows and costs, thus assuming that the signal timings are known, $\mathbf{g}=\mathbf{g}_{\mathbf{0}}$, equilibrium assignment can be expressed by fixed-point models by combining the arc cost function and the arc flow function $[10,11]$ :

$$
\begin{aligned}
& \mathbf{c}^{*}=\mathbf{c}\left(\mathbf{f}^{*}, \mathbf{g}=\mathbf{g}_{\mathbf{0}}\right) \in R^{n} ; \\
& \mathbf{f}^{*}=\mathbf{f}\left(\mathbf{c}^{*}\right) \in S_{f} \subseteq R^{n} ;
\end{aligned}
$$

In the following the arc cost function is assumed continuous and continuously differentiable w.r.t. arc flows, $\mathbf{f}$, with Jacobian matrix $\mathbf{J}_{\mathbf{c}}\left(\mathbf{f}, \mathbf{g}=\mathbf{g}_{\mathbf{0}}\right)=$ $=\mathbf{J a c}\left[\mathbf{c}\left(\mathbf{f}, \mathbf{g}=\mathbf{g}_{\mathbf{0}}\right)\right]_{\mathbf{f}}$. For given signal timings $\mathbf{g}=\mathbf{g}_{\mathbf{0}}$, w.r.t. arc flows, $\mathbf{f}$, the arc cost function may be assumed: monotone strictly increasing (or simply monotone 
increasing), say with (semi-)positive definite (for real vectors) Jacobian $\mathbf{J}_{\mathbf{c}}(\mathbf{f})$, and possibly with symmetric Jacobian $\mathbf{J}_{\mathbf{c}}(\mathbf{f})$, as it occurs for separable cost functions.

Other equivalent models can be formulated w.r.t. path variables:

$$
\mathbf{f}^{*}=\mathbf{f}\left(\mathbf{c}\left(\mathbf{f}^{*}, \mathbf{g}=\mathbf{g}_{\mathbf{o}}\right)\right) \in S_{f}
$$

Sufficient conditions for existence of solutions can be easily derived, through Brouwer theorem, requiring that both the arc cost function and the arc flow function are continuous (and the network is connected).

Assuming that the arc flow function is monotone decreasing, as for invariant probabilistic path choice functions, if the arc cost function is monotone strictly increasing uniqueness is assured; if the Jacobian matrices of both the arc flow function, $\mathbf{f}(\mathbf{c})$, and the arc cost function, $\mathbf{c}\left(\mathbf{f}, \mathbf{g}=\mathbf{g}_{\mathbf{0}}\right)$, are meaningful, uniqueness is granted by positive definite $\mathbf{J}_{\mathbf{c}}\left(\mathbf{f}, \mathbf{g}=\mathbf{g}_{\mathbf{0}}\right)$ and negative semi-definite $\mathbf{J}_{\mathbf{f}}(\mathbf{c})$.

Considering static flows (where flows do not change from one day to another one and are assumed time-independent) three cases can be considered: (I) signal setting with fixed flows $\left(\mathbf{f}_{\mathbf{o}}\right)$, (II) signal setting with feasible flows and (III) signal setting with equilibrium assignment.

I. For a given Fixed Flow $(\mathrm{FF})$ arc vector, $\mathbf{f}=\mathbf{f}_{\mathbf{0}}$, most signal setting methods based on arrival flows can formally be expressed as:

$$
\mathbf{g}^{\mathrm{FF}}=\operatorname{argopt}_{\mathbf{g} \in S g} z\left(\mathbf{g}, \mathbf{f}=\mathbf{f}_{\mathbf{0}}\right)
$$

where $z\left(\mathbf{g}, \mathbf{f}=\mathbf{f}_{\mathbf{0}}\right)$ is an objective function to be optimized, such as the total cost or total delay to be minimized:

$z\left(\mathbf{g}, \mathbf{f}=\mathbf{f}_{\mathbf{0}}\right)=\mathbf{f}_{\mathbf{0}}^{\top} \mathbf{c}\left(\mathbf{f}=\mathbf{f}_{\mathbf{0}}, \mathbf{g}\right)$

II. Global Optimization (GO) model for signal setting, where arc flow vectors are assumed belonging to the feasible arc flow set can formally be expressed as:

$$
\left(\mathbf{g}^{\mathrm{GO}}, \mathbf{f}^{\mathrm{GO}}\right)=\operatorname{argopt}_{\mathbf{g} \in S g, \mathbf{f} \in S f} z(\mathbf{g}, \mathbf{f})
$$

III. Equilibrium constrained Global Optimization (EGO) models are extension of (unconstrained) global optimization models (7):

$$
\begin{gathered}
\left(\mathbf{g}^{\mathrm{EGO}}, \mathbf{f}^{\mathrm{EGO}}\right)=\operatorname{argopt}_{\mathbf{g} \in S g, \mathbf{f} \in S f} z(\mathbf{g}, \mathbf{f}) \\
\text { w.r.t. } \mathbf{f}-\mathbf{f}(\mathbf{c}(\mathbf{f}, \mathbf{g}))=0
\end{gathered}
$$

\subsection{Day-to-day dynamic flows}

This section formally introduces signal setting with (day-to-day) dynamic process assignment after [12], where evolution over day $t$ is explicitly modelled. Congestion is simulated assuming that arc costs depend on arc flows $\mathbf{f}^{\mathbf{t}}$ and on signal timings $\mathbf{g}$, through the arc cost function:

$$
\mathbf{c}=\mathbf{c}\left(\mathbf{f}^{\mathbf{t}}, \mathbf{g}\right)
$$

The arc flow function can be defined:

$$
\mathbf{f}=\mathbf{f}\left(\mathbf{x}^{\mathrm{t}}\right)=\sum_{\mathrm{i}} d_{i} \mathbf{B}_{\mathrm{i}} \mathbf{p}_{\mathrm{i}}\left(-\mathbf{B}_{\mathrm{i}}^{\top} \mathbf{x}^{\mathrm{t}}\right) \in S_{f}
$$

The specification of a dynamic process requires the explicit modelling of user learning and forecasting and user choice updating behaviour. A quite simple but effective model based on exponential smoothing is presented assuming that 
signal timings are known, $\mathbf{g}=\mathbf{g}_{\mathbf{0}}$. Arc forecasted costs depend on yesterday actual and forecasted costs, through the cost updating recursive equation:

where $\beta \in] 0,1]$.

$$
\mathbf{x}^{\mathrm{t}}=\beta \mathbf{c}\left(\mathbf{f}^{\mathrm{t}-1}, \mathbf{g}=\mathbf{g}_{\mathbf{0}}\right)+(1-\beta) \mathbf{x}^{\mathrm{t}-1}
$$

Moreover, each day users may review yesterday choice with a fixed probability, and their reviewing behaviour can be simulated with an exponential smoothing filter, through the flow updating recursive equation:

$$
\mathbf{f}^{\mathbf{t}}=\alpha \mathbf{f}\left(\mathbf{x}^{\mathbf{t}}\right)+(1-\alpha) \mathbf{f}^{\mathbf{t}-1}
$$

where $\alpha \in] 0,1]$.

The recursive equations (12) and (13) define a deterministic process model for demand assignment to a transportation network. For given signal timings, $\mathbf{g}=$ $\mathbf{g}_{\mathbf{0}}$, the state at day $\mathrm{t}$ is defined by the vectors of arc anticipated costs and arc flows, $\left(\mathbf{x}^{\mathrm{t}}, \mathbf{f}^{\mathrm{t}}\right)$, belonging to state space given by $R^{n} ; \times S_{f}$.

Fixed-points states, where the evolution over time of the system stops (even though they may not be attractors), are given by:

$$
\left(\mathbf{x}^{\mathrm{t}}, \mathbf{f}^{\mathrm{t}}\right)=\left(\mathbf{x}^{\mathrm{t}-1}, \mathbf{f}^{\mathrm{t}-1}\right)=\left(\mathbf{x}^{*}, \mathbf{f}^{*}\right)
$$

This condition combined with equations (12) and (13) yields:

$$
\begin{gathered}
\mathbf{x}^{*}=\mathbf{c}\left(\mathbf{f}^{*}, \mathbf{g}=\mathbf{g}_{\mathbf{0}}\right) \\
\mathbf{f}^{*}=\mathbf{f}\left(\mathbf{x}^{*}\right)
\end{gathered}
$$

Assuming that the arc flow function has a symmetric negative semi-definite Jacobian, $\mathbf{J}_{\mathbf{f}}(\mathbf{c})$, as it occurs for invariant choice functions, if Jacobian, $\mathbf{J}_{\mathbf{c}}(\mathbf{f})$, of arc cost function is symmetric positive definite, then matrix $\mathbf{J}_{\mathbf{c}}\left(\mathbf{f}^{\mathbf{t}}, \mathbf{g}=\mathbf{g}_{\mathbf{0}}\right) \cdot \mathbf{J}_{\mathbf{f}}\left(\mathbf{x}^{\mathbf{t}}\right)$ has only non-positive real eigenvalues, $\omega_{\mathrm{a}}=\omega_{\mathrm{a}}\left(\mathbf{f}^{\mathrm{t}}, \mathbf{x}^{\mathrm{t}}\right)=\operatorname{Re}\left(\omega_{\mathrm{a}}\right)$. In this case a fixed-point $\left(\mathbf{x}^{*}, \mathbf{f}^{*}\right)$ is (locally) stable if each eigenvalue $\omega_{\mathrm{a}}{ }^{*}=\omega_{\mathrm{a}}\left(\mathbf{f}^{*}, \mathbf{x}^{*}\right)$ of matrix $\mathbf{J}_{\mathbf{c}}\left(\mathbf{f}^{*}, \mathbf{g}=\mathbf{g}_{\mathbf{0}}\right) \cdot \mathbf{J}_{\mathbf{f}}\left(\mathbf{x}^{*}\right)$, computed at the fixed-point state, meets the following condition

$$
\left|\omega_{\mathrm{a}}\left(\mathbf{f}^{*}, \mathbf{x}^{*}\right)\right|=-\operatorname{Re}\left(\omega_{\mathrm{a}}^{*}\right)<\omega_{\mathrm{o}} \forall a
$$

where $\omega_{0}=(1+2((1-\alpha)+(1-\beta)) /(\alpha \beta))$.

Equilibrium stability may be addressed within models for (global) optimization with equilibrium assignment by including further constraints expressing stability conditions depending on assumptions about the Jacobian matrix $\mathbf{J}_{\mathbf{c}}\left(\mathbf{f}, \mathbf{g}=\mathbf{g}_{\mathbf{0}}\right)$ of arc cost function. Thus, a model for global optimization of signal timing with stable equilibrium constraints (SEGO) may be specified as follows:

$$
\begin{gathered}
\left(\mathbf{g}^{\mathrm{SEGO}}, \mathbf{f}^{\mathrm{SEGO}}\right)=\operatorname{argopt} \mathbf{g}_{\mathbf{g} \in S g, \mathbf{f} \in S f} z(\mathbf{g}, \mathbf{f}) \\
\text { w.r.t. } \mathbf{f}-\mathbf{f}(\mathbf{c}(\mathbf{f}, \mathbf{g}))=0 \\
\operatorname{MAX}_{\mathrm{a}}\left|\omega_{\mathrm{a}}(\mathbf{f}, \mathbf{c}(\mathbf{f}, \mathbf{g}))\right|<\omega_{\mathrm{o}}
\end{gathered}
$$

The evaluation of (20) to a large scale network is time consuming. It is useful to note that the maximum modulus among all the eigenvalues of a matrix is always less than (or equal to) any matrix norm and for transportation networks the Frobenius norm provides a very tight approximation and at the same time can easily be computed; hence (20) can be approximated as:

$$
\Delta \omega(\mathbf{f}, \mathbf{g})=\left\|\mathbf{J}_{\mathbf{c}}(\mathbf{f}, \mathbf{g}) \cdot \mathbf{J}_{\mathbf{f}}(\mathbf{c}=\mathbf{c}(\mathbf{f}, \mathbf{g}))\right\|_{F}-\omega_{\mathrm{o}}<0
$$




\section{Proposed procedure}

The design procedure (figure 1) proposed in this paper aims at the solution of signal setting with stability constraints derived from a day-to-day dynamic model. The procedure generates and analyses potential solutions in order to optimize an objective function. It is made up by two main interacting subprocedures: an optimization module, based on a genetic algorithm, which provides new solution to be add to the population of solutions; an assignment module, based on a fixed-point model (section 2), which provides flows and objective function values for each generated solutions; the objective function also includes the stability constraints through a penalty function.

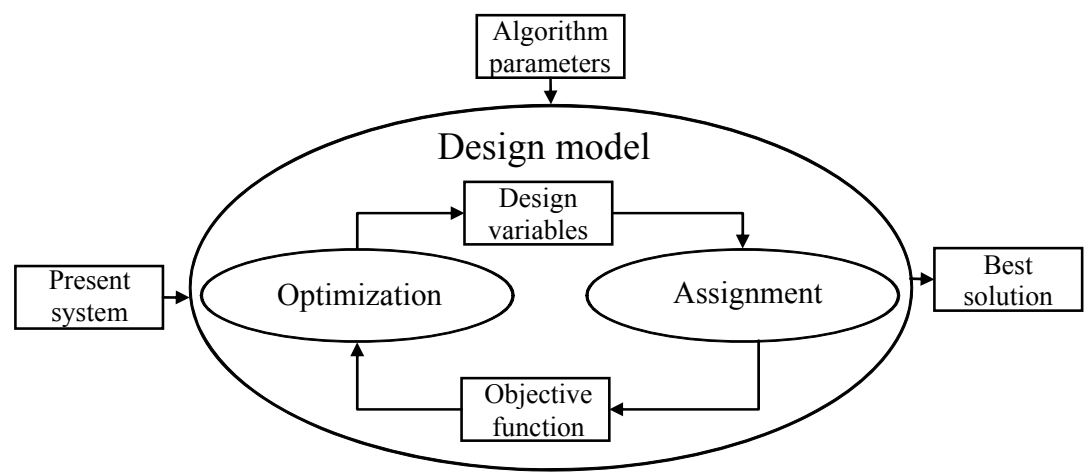

Figure 1: Design procedure.

According to the optimization genetic algorithm each solution vector $\mathbf{g}$ is described by a chromosome (figure 2 ) with three sections. The first has one entry containing the common value of cycle time, useful for cycle evaluation relative to each junction. The second has a number of entries equal to the number of junctions and each of them being the integer multiple, $s_{M u}$, of the cycle time of junction $u$ w.r.t the common cycle time, $C_{B}$. The thirty contains the ratios, $\zeta_{\mathrm{j}}^{\mathrm{u}}$, between the time of each stage $j$ of junction $u$ and the cycle time for the same junction; in each junction, the number of these entries is equal to the number of stages less 1. (The structure can be extended to include offsets).

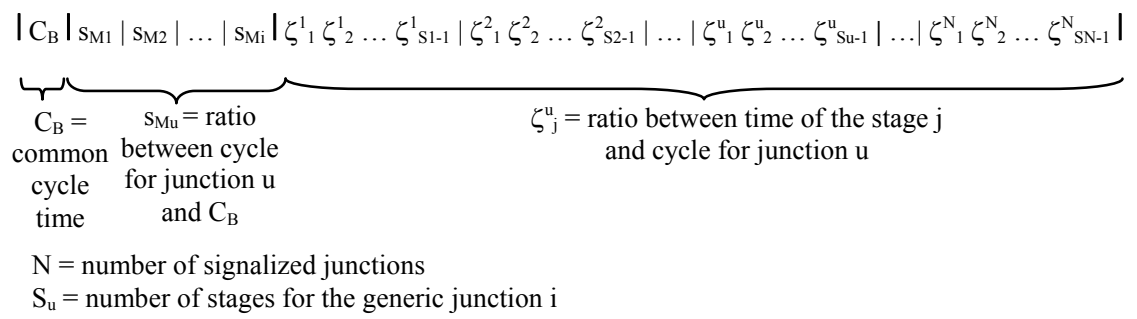

Figure 2: Structure of the chromosome. 
The optimization genetic algorithm (figure 3) starts with an initial set of solutions (POP), grouped into a randomly generated population (Pop_G). A new population Pop_A is generated. Afterwards, population evolves through three operators (or evolution functions), which are sequentially applied. Reproduction consists in selecting a sub-set of solutions from the population, the best will likely survive and create new offspring (Pop_R). Crossover selects genes from parent chromosomes and creates new offspring (Pop_C). Then, the mutation takes place and it changes randomly the new offspring, in order to prevent all solutions in the population falling into the local optimum of solved problems (Pop_M). Once a new set of solutions is generated, a stopping test evaluates whether a new iteration must be performed or not, according to some stopping criteria. At the end of each iteration, the best solution replaces the current project.

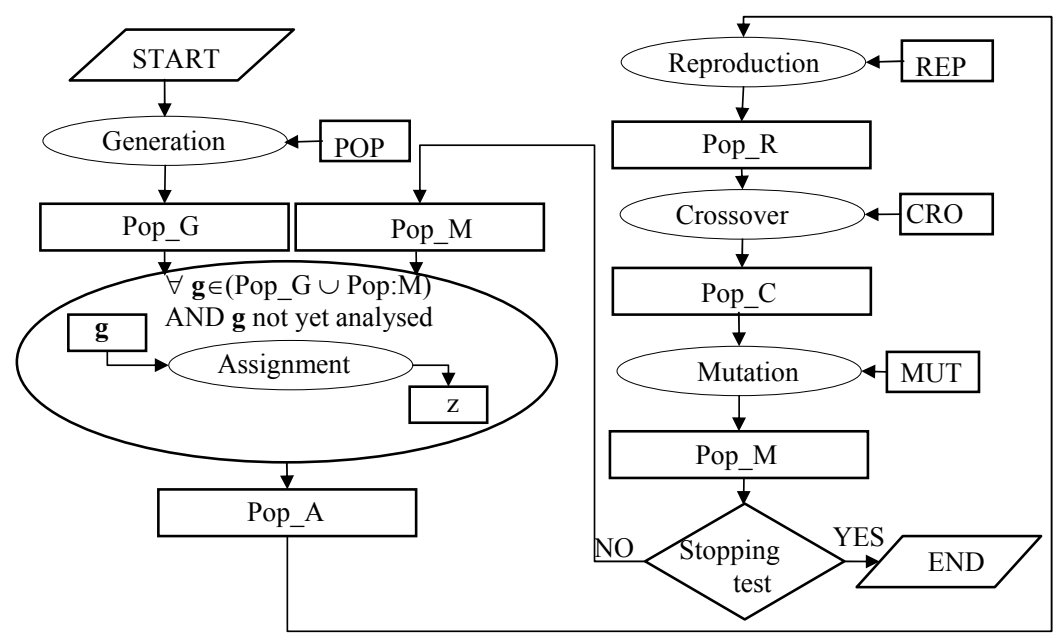

Figure 3: $\quad$ Structure of the solution generator procedure.

In each stage, new solutions are evaluated with the assignment procedure for the estimation of objective function, $z$. The stability constraint for penalizing those solutions that does not guarantee the equilibrium stability through a poor value of the objective function is activated.

The procedure has two types of input relative to the transport system models and to the algorithm parameters.

The transportation system is specified with travel demands models (that represent how users behaviour changes with users cost), transport supply models (that represent how users cost changes with traffic flow) and demand-supply interaction model. In the supply models the dependence between signal setting and traffic flows is also included. The models are relative to the present configuration of supply facilities and services and user behaviour.

The genetic algorithm parameters are the population size (POP), the crossover (CRO) and mutation (MUT) rates, the reproduction parameter (REP), and the 
convergence test parameter. Constraints on the minimum and maximum cycle length, and minimum green time are also considered. The transport model gives for each solution $\mathbf{g}$, a global network indicator, which represents the objective function, including a penalty term for the stability constraint, then, the current optimal solution updated $\mathbf{g}^{*}$. The algorithm stops when the (pre-fixed) maximum number of iterations without improving the value of the objective function is reached. Output is the current optimal vector $\mathbf{g}^{*}$.

\section{Application}

The procedure has been applied to the network of Crotone, a city in the southern Italy, containing 15 centroids, 157 nodes and 432 arcs, 88 OD pairs. Seven signalized junctions have been identified.

Each street link, ending in a signalized junction, is modelled through a running arc and possibly several parallel access arcs. For the run arcs, the Davidson hyperbolic function with a linear approximation of 0.75 for the flowcapacity ratio has been adopted; for access arcs, the Webster function with a linear approximation 0.40 for the flow-capacity ratio has been adopted.

The equilibrium flow vector is obtained with an user equilibrium assignment with (probabilistic) C-Logit route choice model and explicit path enumeration. A (scale) dispersion parameter, $\theta$, equal to 2 hours has been considered.

In relation to the solution generator, the parameters adopted are:

- crossover rate 0.4 ;

- mutation rate 0.4 ;

- maximum number of iterations 10 ;

- population numerousness 30 chromosomes.

The objective function $z$ is evaluated considering the sum of the delays at the designed junctions.

The constraints are:

- cycle times must be in the range [27 sec, $120 \mathrm{sec}]$;

- each stage must be no less than $7 \mathrm{sec}$;

- user behaviour is expressed with equilibrium condition (19);

- stability of equilibrium is expressed by stability condition (21).

In order to define the best genetic parameters, in the table 1 the procedure is tested for some values $(0,2,0,4,0,6,0,8)$ of the crossover and mutation rate $(\mathrm{d}=$ $\left.5300 \mathrm{veic} / \mathrm{h} ; \theta=2 \mathrm{~h} ; \omega_{0}(\alpha, \beta)=9.5\right)$. The best value in term of convergence is for crossover rate and mutation rate equal to 0,4 .

Table 1: Sensibility of the algorithm in relation to the crossover and mutation rate.

\begin{tabular}{ccccccc}
\hline Crossover & Mutation & \multicolumn{2}{c}{ Total delay $($ veic $h / h)$} & & \multicolumn{2}{c}{$\Delta \omega$} \\
\cline { 3 - 4 } \cline { 6 - 7 } rate & rate & actual & optimal & & actual & Optimal \\
\hline 0,8 & 0,8 & 319 & 69 & & $-4,014$ & $-4,008$ \\
0,6 & 0,6 & 319 & 71 & & $-4,014$ & $-4,008$ \\
0,4 & 0,4 & 319 & 58 & & $-4,014$ & $-4,008$ \\
0,2 & 0,2 & 319 & 65 & & $-4,014$ & $-4,008$ \\
\hline
\end{tabular}


The problem has been solved for different values of total travel demand for the morning peak hour: 4240, 5300 (actual demand), $6360 \mathrm{veic} / \mathrm{h}$. It is assumed for the simulation that $\alpha=0.70$ (parameters for choice updating) and $\beta=0.5$ (parameter for cost updating) which give $\omega_{0}(\alpha, \beta)=5.5$. The procedure is started assuming the actual signal timings as current optimal solution; it is worth noting that this solution could not be stable depending on the value of $\omega_{0}(\alpha, \beta)$.

Considering the actual demand $(5300 \mathrm{veic} / \mathrm{h})$, the proposed procedure, after few iterations, provides a stable $(\Delta \omega=-0.001)$ optimal solution, with a $35 \%$ reduction of total delay with respect to the actual (stable) solution, as shown in figure 4 by black triangles. This figure also shows results of increasing $\omega_{0}(\alpha, \beta)$ from 5.5 to 9.5 ; a new solution with a further reduction of total delay is obtained (black circles); as expected since the constraint has been relaxed stability condition is satisfied $(\Delta \omega=-4.014)$.

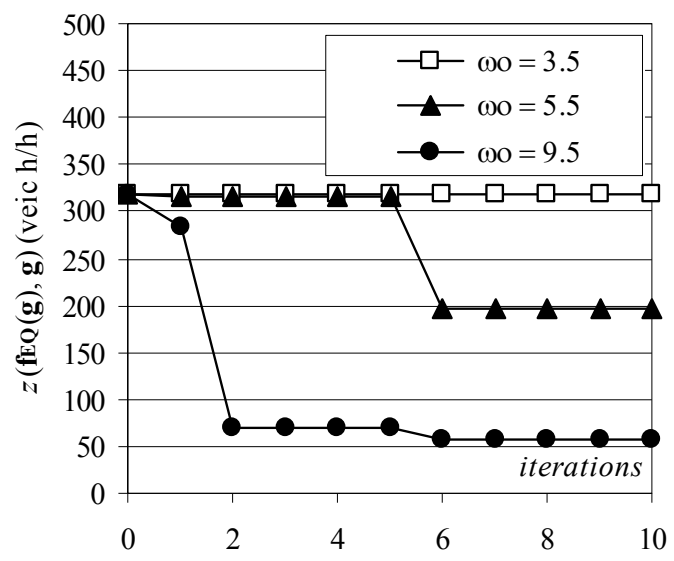

Figure 4: Results of the optimization procedure for demand $=5300$ for different values of $\omega_{0}$.

On the other hand, after decreasing $\omega_{0}(\alpha, \beta)$ from 5.5 to 3.5 , the actual solution is not stable (showing $\Delta \omega=1.964$ ) and the current solution is not updated, since no other stable solution can be found (blank squares). This example supports the theoretical conclusion that existence of a solution satisfying both equilibrium and stability constraints may not exist. This case becomes more likely after any increase of demand. Effects of changes of demand are briefly discussed below.

It is worth noting that if a unique solution to equilibrium assignment exists $\mathbf{f}^{*}$, it turns out to be a function of the signal timings, $\mathbf{f}^{*}=\mathbf{f}_{\mathrm{EQ}}(\mathrm{g})$.

Figure 5 shows the effect of reduction of demand from 5300 to 4240. In this case too, for $\omega_{0}(\alpha, \beta)$ equal to 5.5 or 9.5 a stable solution better than the actual one may be found, whilst no stable solution may be found for $\omega_{0}(\alpha, \beta)$ equal to 3.5 . 
782 Sustainable Development and Planning V

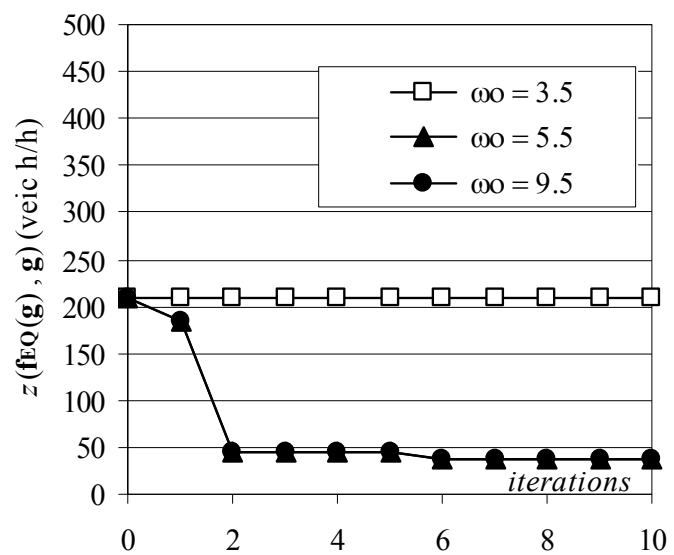

Figure 5: Results of the optimization procedure for demand $=4240$ for different values of $\omega_{0}$.

Figure 6 shows the effect increase of demand from 5300 to 6360. In this case, a stable solution better than the actual one may be found for $\omega_{0}(\alpha, \beta)$ equal to 9.5 only, whilst no stable solution may be found for $\omega_{0}(\alpha, \beta)$ equal to 3.5 or 5.5.

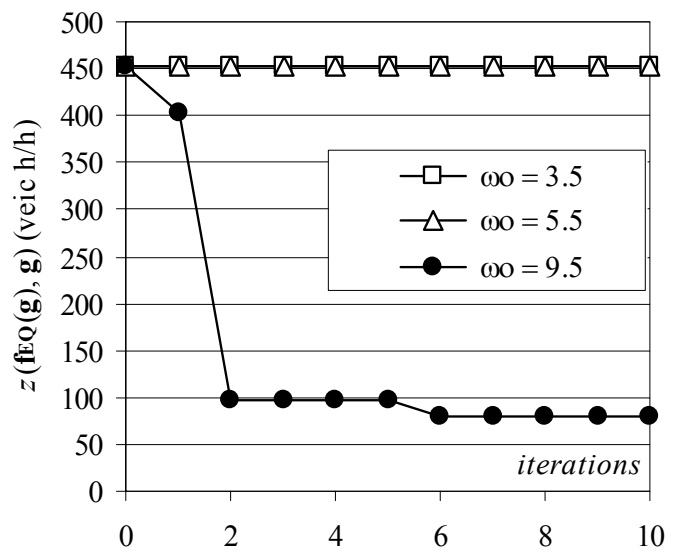

Figure 6: Results of the optimization procedure for demand $=6360$ for different values of $\omega_{0}$.

\section{Conclusions}

In this paper the problem of signal setting with (day-to-day) dynamic process assignment has been described. This problem seems relevant since optimization of signal timings under equilibrium assumptions not guarantee that an effective 
solution is obtained as the obtained solution may not satisfy stability conditions (as those described in the paper).

A heuristic solution procedure has also been specified on genetic algorithm for the solution generation. Results of an application to a real scale network (with C-Logit route choice model) have also been reported. These results confirm that an increase of demand may make stability less likely. Some values for procedure parameters are tested.

Several issues seem worth of further research analysis. Existence and uniqueness of solution of proposed models could be investigated. Other signal variables may be considered such as offsets, possibly together with street directions. Solution procedures based on other heuristics may be tested and compared with the proposed procedure. The calibration of all the parameters (model and procedure parameters) is a relevant issue still open apart from very few papers.

\section{Acknowledgements}

Partially supported under UNISA local grant n. ORSA091208 financial year 2009, local grant n. ORSA107325 financial year 2010, and PRIN national grants n. 2007R9CSXY_003 at UNIRC and 2007R9CSXY_004 at UNISA financial year 2007.

\section{References}

[1] Cascetta E. (2009) Transportation Systems Analysis. Springer.

[2] Cantarella G. E., Vitetta A. (2006) The multi-criteria road network design problem in an urban area. Transportation, 33, pp. 357-588.

[3] Russo F., Vitetta A. (2006) A Topological Method to Choose Optimal Solutions after Solving the Multi-criteria Urban Road Network Design Problem. Transportation, 33, pp. 347-370.

[4] Cantarella G. E., Pavone G., Vitetta A. (2006) Heuristic for urban road network design: lane layout and signal setting. European Journal of Operational Research, 175, pp. 1682-1695.

[5] Papageorgiou M. (editor) (1991) Concise Encyclopedia of Traffic and Transportation. Pergamon Press.

[6] Allsop R.E. (1974) Some possibilities for using traffic control to influence trip destinations and route choice. Proceedings of the Sixth International Symposium on Transportation and Traffic Theory. Buckley ed., Amsterdam, Elsevier, pp. 345-374.

[7] Russo F. \& Vitetta A. (2006) Risk evaluation in a transportation system. International Journal of Sustainable Development and Planning, 1 (2), pp. 170-191.

[8] Marcianò A., Musolino G., Vitetta A. (2010) Signal setting design on a road network: application of a system of models in evacuation conditions. Proceedings of Risk Analysis VII. WIT Press. Edited by C. Brebbia, pp. 443, 454. 
784 Sustainable Development and Planning V

[9] Cantarella G. E, Velonà P., Vitetta A. (2010) Signal setting with demand assignment: global optimization with equilibrium stability constraints. The Third International Symposium on Dynamic Traffic Assignment, 29-31 July, 2010, Takayama, Japan.

[10] Daganzo C.F. (1983) Stochastic Network Equilibrium with Multiple Vehicle Types and Asymmetric, Indefinite Link Cost Jacobians. Transportation Science, 17, pp. 282-300.

[11] Cantarella G.E. (1997) A General Fixed-Point Approach to Multi-Mode Multi-User Equilibrium Assignment with Elastic Demand. Transportation Science, 31, pp. 107-128.

[12] Cantarella G.E. (2009) Signal setting with dynamic process assignment. New Developments in Transport Planning: Advances in Dynamic Traffic Assignment. Immers L.H., Tampere C.M.J., Viti F. (eds), 2009. Edward Elgar, Cheltenham, UK - Northampton, MA, USA. 